\title{
Resveratrol exerts anti-obesity effects via mechanisms involving down-regulation of adipogenic and inflammatory processes in mice
}

\author{
K. Soyoung, J. Yoojeong, C. Youngshim and P. Taesun \\ Department of Food and Nutrition, Yonsei University, 262 Seongsanno Seodaemun-gu, Seoul 120-749, Korea
}

Resveratrol is a natural polyphenolic stilbene derivative found in a variety of edible fruits, including nuts, berries, and grape skin ${ }^{(1)}$. Although resveratrol has been suggested to improve thermogenesis in the brown adipose tissues of obese animals, there have been no reports on the anti-adipogenic and anti-inflammatory effects of resveratrol in the white adipose tissues of obese animals. The primary aim of this study was to investigate whether resveratrol attenuates high-fat diet (HFD)-induced adipogenesis and inflammation in the epididymal fat tissues of mice and to explore the underlying mechanisms involved in this attenuation. Male C57BL/6N mice were fed a normal diet ( $n$ 10), HFD ( $n$ 10) or a $0.4 \%$ resveratrol-supplemented diet (RSD, $n$ 10) for 10 weeks. The plasma and hepatic lipid levels were determined by enzymatic kits, and the adipose tissue gene and protein expression levels were analysed via RT-PCR and Western blotting, respectively. These data were analysed using ANOVA with Duncan's multiple-range tests.

In comparison with HFD mice, mice fed with a RSD showed significantly $(P<0.05)$ lower body weight gain $(-48 \%)$, visceral fat-pad weights $(-58 \%)$ and plasma levels of TAG, FFA, total cholesterol, glucose, TNF $\alpha$ and MCP1. Resveratrol significantly $(P<0.05)$ reversed the HFD-induced up-regulation of galanin-mediated signalling molecules (GalR1/2, PKC $\delta$, Cyc-D, E2F1 and p-ERK) and key adipogenic genes (PPAR $\gamma 2$, C/EBP $\alpha$, SREBP-1c, FAS, LPL, aP2 and leptin) in the epididymal adipose tissues of mice. Furthermore, resveratrol significantly $(P<0.05)$ attenuated the HFD-induced up-regulation of pro-inflammatory cytokines $(\mathrm{TNF} \alpha$, IFN $\alpha$, IFN $\beta$ and IL-6) and their upstream signalling molecules (TLR2/4, MyD88, Tirap, TRIF, TRAF6, IRF5, p-IRF3 and NF- $\kappa B$ ) in the adipose tissues of mice. The results of this study suggest that resveratrol inhibits visceral adipogenesis by suppressing the galanin-mediated adipogenesis signalling cascade. It may also attenuate cytokine production in the adipose tissue by repressing the TLR2- and TLR4-mediated proinflammatory signalling cascades in HFD-fed mice (Fig. 1). The results of this study suggest that resveratrol inhibits visceral adipogenesis by suppressing the galanin-mediated adipogenesis signalling cascade. It may also attenuate cytokine production in the adipose tissue by repressing the TLR2- and TLR4-mediated pro-inflammatory signalling cascades in HFD-fed mice.

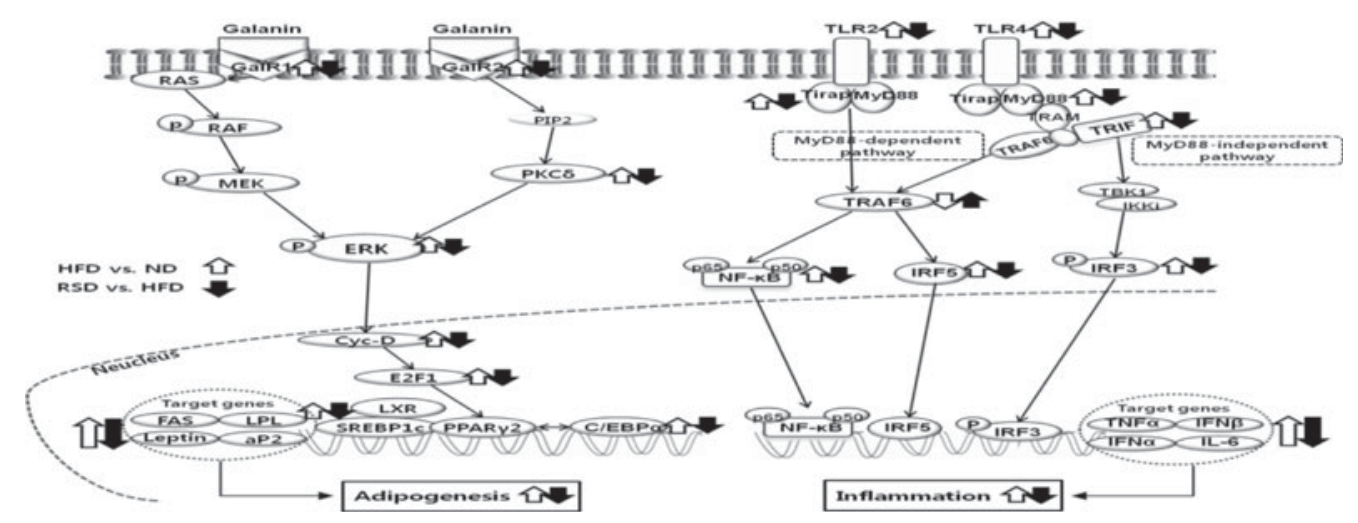

Fig. 1. The possible molecular mechanisms of dietary resveratrol in attenuating adipogenesis and inflammation induced by HFD.

1. Ren S \& Lien EJ (1997) Prog Drug Res 48, 147-171. 